\title{
A Hamiltonian-based solution to the mixed sensitivity optimization problem for stable pseudorational plants
}

\author{
Kenji Kashima $^{\mathrm{a}, *}$, Hitay Özbay ${ }^{\mathrm{b}, 1}$, Yutaka Yamamoto ${ }^{\mathrm{a}}$ \\ ${ }^{a}$ Department of Applied Analysis and Complex Dynamical Systems, Graduate School of Informatics, Kyoto University, Kyoto 606-8501, \\ Japan \\ ${ }^{\mathrm{b}}$ Department of Electrical and Electronics Engineering, Bilkent University, Bilkent, Ankara TR-06800, Turkey
}

Received 30 December 2004; accepted 26 March 2005

Available online 10 May 2005

\begin{abstract}
This paper considers the mixed sensitivity optimization problem for a class of infinite-dimensional stable plants. This problem is reducible to a two- or one-block $H^{\infty}$ control problem with structured weighting functions. We first show that these weighting functions violate the genericity assumptions of existing Hamiltonian-based solutions such as the well-known Zhou-Khargonekar formula. Then, we derive a new closed form formula for the computation of the optimal performance level, when the underlying plant structure is specified by a pseudorational transfer function.
\end{abstract}

(c) 2005 Elsevier B.V. All rights reserved.

Keywords: Pseudorational transfer function; Infinite-dimensional systems; Mixed sensitivity optimization; $H^{\infty}$ control; Skew-Toeplitz approach

\section{Introduction}

Since mid-1980s various methods have been developed for the $H^{\infty}$ control of infinite-dimensional systems. In particular, for the one-block problem of finding

$\rho_{\mathrm{opt}}:=\inf _{Q \in H^{\infty}}\|W-m Q\|_{\infty}$

\footnotetext{
* Corresponding author. Tel.: +81757535904 ; fax: +81757535517 .

E-mail addresses: kashima@acs.i.kyoto-u.ac.jp (K. Kashima), ozbay@ee.eng.ohio-state.edu (H. Özbay), yy@i.kyoto-u.ac.jp (Y. Yamamoto).

${ }^{1}$ On leave from The Ohio State University, USA.
}

with $m$ being an pure time delay, and $W$ given as a strictly proper rational function, a closed form expression has been obtained by Zhou and Khargonekar [15]. The formula has been extended to more general cases in $[3,8,10,14]$ : Let $H_{\rho, W}$ be the Hamiltonian matrix associated with $W$ and $\rho$ :

$H_{\rho, W}:=\left[\begin{array}{cc}A & B B^{\mathrm{T}} / \rho \\ -C^{\mathrm{T}} C / \rho & -A^{\mathrm{T}}\end{array}\right]$,

where $(A, B, C)$ is a minimal realization of $W$. Suppose that $m$ is analytic on the set of eigenvalues of $H_{\rho, W}$. Then the optimal sensitivity $\rho_{\text {opt }}$ is the maximal $\rho$ such that $\operatorname{det}\left(\left.\tilde{m}\left(H_{\rho, W}\right)\right|_{22}\right)=0$ where $\left.M\right|_{22}$ denotes 
the (2,2)-block of matrix $M$ partitioned accordingly to (1). Recently, in [7], it was shown that when a plant is pseudorational [12], $\tilde{m(}\left(H_{\rho, W}\right)$ is easily obtainable without numerical computations of poles and zeros of the transfer function; see Lemma 2.

In this paper, we consider the mixed sensitivity optimization problem

$\gamma_{\mathrm{opt}}:=\inf _{C \text { stabilizing } P}\left\|\left[\begin{array}{c}W_{S}(1+P C)^{-1} \\ W_{t} P C(1+P C)^{-1}\end{array}\right]\right\|_{\infty}$,

where $W_{s}$ and $W_{t}$ are rational weights, and $P$ is a stable pseudorational plant. This problem is known to be a typical two-block problem, for which a Hamiltonianbased formula is obtained [4]. However, this result is not directly applicable, since a "generic" assumption of the formula is almost always violated [6]. In view of this, we derive a Hamiltonian-based formula for the optimal mixed sensitivity computation, by reducing this structured two-block problem to a one-block problem. This result can be viewed as an extension of the Zhou-Khargonekar formula to a specifically structured one-block problem.

The paper is organized as follows: in the next section we review some preliminary results on pseudorational systems. In Section 3, we briefly summarize the observations made in [7] and state drawbacks in more precise terms. In Section 4, we derive a Hamiltonianbased solution for the structured one-block problem. A numerical example is given in Section 5, and concluding remarks are made in the last section.

\section{Notation and Convention}

As usual, $H^{p}$ and $H_{-}^{p}$ denote the Hardy $p$-spaces on the open right- and left-half complex planes, respectively. Let $\tilde{q}(s):=q(-s)$. For an inner function $m$, let $H(m)$ be the orthogonal complement of $m H^{2}$ in Hilbert space $H^{2}$. It is known [5] that

$H(m)=\left\{x \in H^{2}: \tilde{m x} \in H_{-}^{2}\right\}$.

For a given distribution (in the sense of Schwartz [9]) $\alpha, \operatorname{supp} \alpha$ denotes its support [9], and

$$
\begin{aligned}
& \ell(\alpha):=\inf \{t: t \in \operatorname{supp} \alpha\}, \\
& r(\alpha):=\sup \{t: t \in \operatorname{supp} \alpha\} .
\end{aligned}
$$

Let $\mathscr{E}^{\prime}\left(\mathbb{R}_{-}\right)$denote the space of distributions having compact support in $(-\infty, 0] . \mathscr{D}_{+}^{\prime}(\mathbb{R})$ is the space of distributions having support bounded on the left. Clearly $\mathscr{E}^{\prime}\left(\mathbb{R}_{-}\right) \subset \mathscr{D}_{+}^{\prime}(\mathbb{R})$. If a distribution $\alpha$ is Laplace transformable, its Laplace transform is denoted by $\hat{\alpha}(s)$.

\section{Preliminaries on pseudorational systems}

In this section we review certain basic facts on pseudorational systems. This class of systems has been introduced in the late 1980's, and plays a crucial role in realization, modeling, and control of infinitedimensional systems, especially delay-differential systems [12,13]:

Definition 1. Let $f$ be a distribution having support in $[0, \infty)$. It is said to be pseudorational if there exist $q, p \in \mathscr{E}^{\prime}\left(\mathbb{R}_{-}\right)$such that

(1) $q^{-1}$ exists over $\mathscr{D}_{+}^{\prime}(\mathbb{R})$,

(2) $\operatorname{ord} q^{-1}=-\operatorname{ord} q$,

(3) $f$ can be written as $f=q^{-1} * p$,

where $q^{-1}$ is taken with respect to convolution and ord $q$ denotes the order of a distribution $q$ [9].

If $f$ is pseudorational, its associated transfer function $\hat{f}$ is also said to be pseudorational. From the Paley-Wiener-Schwartz theorem [9], in the Laplace domain, every pseudorational transfer function is a ratio of entire functions of exponential type-the simplest extension of rational functions. For a stable pseudorational plant $P$, even if $P$ is not necessarily inner,

$\rho_{\text {opt }}:=\inf _{Q \in H^{\infty}}\|W-P Q\|_{\infty}$

can be computed by the following:

Lemma 2 (Kashima and Yamamoto [7]). Suppose that $P$ can be factorized as $\hat{p}_{1} \hat{p}_{2} / \hat{q}$ with $q, p_{1}, p_{2} \in$ $\mathscr{E}^{\prime}\left(\mathbb{R}_{-}\right)$such that $\hat{q}^{-1}, \hat{p}_{1}^{-1}, \mathrm{e}^{r\left(p_{2}\right) s} \hat{p}_{2}^{-1} \in H^{\infty}$, that is, $\hat{p}_{1}$ and $\hat{p}_{2}$ denote the stable and anti-stable parts of the numerator, respectively. Assume also that $1 / \hat{p}_{2}$ is analytic on the set of eigenvalues of $H_{\rho, W}$. Define $L:=-\ell(q)+\ell\left(p_{1}\right)-r\left(p_{2}\right)$ and

$m_{v}(s)=\mathrm{e}^{-L s} \frac{\hat{p}_{2}(s)}{\hat{p}_{2}(s)}$. 
Then $\rho_{\text {opt }}$ in (4) is the maximal $\rho$ that satisfies $\operatorname{det}\left(\left.m_{v} \tilde{(}\left(H_{\rho, W}\right)\right|_{22}\right)=0$.

\section{Mixed sensitivity optimization problem}

\subsection{Two-block problem}

In this section, we show that the weighting functions have a specific structure when we reduce the mixed sensitivity optimization problem to the standard twoblock problem. Throughout the paper the plant $P$ is assumed to be stable. By the Youla parameterization, all stabilizing controllers are given in the form $C=$ $Q(1-P Q)^{-1}, Q \in H^{\infty}$. Hence we obtain

$\gamma_{\mathrm{opt}}=\inf _{Q \in H^{\infty}}\left\|\left[\begin{array}{c}W_{s}(1-P Q) \\ W_{t} P Q\end{array}\right]\right\|_{\infty}$.

First, introduce the following spectral factorization $G$

$G\left(W_{s} W_{s}+W_{t}^{\tau} W_{t}\right) G=1$,

where both $G$ and $G^{-1}$ have no unstable poles. Then it follows that

$L_{1}:=\left[\begin{array}{cc}\left(W_{s} G\right)^{\sim} & \left(-W_{t} G\right)^{\sim} \\ W_{t} G & W_{s} G\end{array}\right], \quad L_{2}:=\left[\begin{array}{cc}m_{d} & 0 \\ 0 & 1\end{array}\right]$

are unitary, where $m_{d}$ is a finite Blaschke product that makes

$W:=m_{d} W_{s}\left(W_{s} G\right)^{r}$

stable [2]. Multiplying (6) by $L_{2} L_{1}$ from the left, we obtain

$\gamma_{\mathrm{opt}}=\inf _{Q \in H^{\infty}}\left\|\left[\begin{array}{c}W-m_{d} P Q \\ V\end{array}\right]\right\|_{\infty}$,

where

$V:=W_{s} W_{t} G$.

Note that both $W$ and $V$ are rational and stable. The problem in the form (9) has been considered in [4], and a solution based on a Hamiltonian related to a realization of $\gamma^{2}-W W-V V$ is derived. It is however assumed in [4] that $V$ and $W$ have no common poles. For arbitrary rational functions $V$ and $W$, this assumption is satisfied generically. However, in the mixed sensitivity problem, the functions $W$ and $V$ need to be in the form (8) and (10). As seen in Appendix, this means that unless $W_{s}$ and $W_{t}$ are chosen in a specific way, $W$ and $V$ will have common poles, i.e., the assumption above is almost always violated.

On the other hand, by (7), (8) and (10), we have

$$
\begin{aligned}
\gamma^{2}-W W-V V & =\gamma^{2}-W_{s} W_{s}^{\tilde{G}} G\left(W_{s} W_{s}^{\tilde{}}+W_{t} W_{t}\right) G^{\sim} \\
& =\gamma^{2}-W_{s} W_{s} \tilde{}
\end{aligned}
$$

Thus (11) may help us to avoid the "genericity" assumption. However, in the argument in [4], it is difficult to introduce such structures on $V$ and $W$, since no relationship between these weights was assumed. In view of this, we reduce the specifically structured two-block problem to a one-block problem to make use of such structures explicitly.

\subsection{Reduction to one-block problem}

Again, applying the standard techniques, see e.g. [2], we now reduce the two-block $H^{\infty}$ problem (9) to a one-block problem. First, suppose that $\gamma>\|V\|_{\infty}$ satisfies $\gamma=\gamma_{\text {opt }}$. Then there exists $Q \in H^{\infty}$ such that

$$
\left|W-m_{d} P Q\right|^{2}+|V|^{2}=\gamma^{2} \text { a.e. }
$$

on the imaginary axis. Here, since $\gamma>\|V\|_{\infty}$, there exists a unique spectral factor $F_{\gamma}$ :

$F_{\gamma}\left(\gamma^{2}-V V\right) F_{\gamma}=1 \quad$ a.e.

where both $F_{\gamma}$ and $F_{\gamma}^{-1} \in H^{\infty}$. Therefore, by defining $W_{\gamma}:=F_{\gamma} W$, we obtain

$\left|W_{\gamma}-m_{d} P Q\right|^{2}=1$ a.e.

on the imaginary axis. Furthermore it is shown [11] that $\gamma_{\text {opt }}$ is given by the maximal $\gamma$ such that 1 is a singular value of the compression operator $W_{c}$ of $W_{\gamma}$ to $H(m)$ defined by

$W_{c}: H(m) \rightarrow H(m): x \mapsto \pi^{m}\left[W_{\gamma} x\right]$,

where $m:=m_{d} m_{v}$ and $\pi^{m}[\cdot]$ denotes the orthogonal projection from $H^{2}$ onto $H(m)$.

Lemma 2 characterizes the singular values of the corresponding compression operator [14], that is, 1 is a singular value of $W_{c}$ if and only if $\left.\tilde{m(}\left(H_{1, W_{\gamma}}\right)\right|_{22}$ is not of full rank, when $m$ is analytic on the set of eigenvalues of $H_{1, W_{\gamma}}$. However, $W_{\gamma}$ and $m_{d}$ have a 
specific structure, and we must be careful in applying Lemma 2. To see this, let us consider the eigenvalues of $H_{1, W_{\gamma}}$. Notice that the eigenvalues of the Hamiltonian matrix $H_{\rho, W}$ coincide with the zeros of $\rho^{2}-W W$. Eqs. (11) and (12) yield

$$
\begin{aligned}
1-W_{\gamma} W_{\gamma} & =\left(\gamma^{2}-V V-W W\right) F_{\gamma}^{\tilde{F}} F_{\gamma} \\
& =\left(\gamma^{2}-W_{s}^{\sim} W_{s}\right) F_{\gamma}^{\sim} F_{\gamma} .
\end{aligned}
$$

Therefore, the eigenvalues of $H_{1, W_{\gamma}}$ arise from those of $H_{\gamma, W_{s}}$ or zeros of $F_{\gamma}$ and $F_{\gamma}$. Unfortunately, the zeros of $F_{\gamma}$ coincide with poles of $m_{d}$; see Appendix and $[6,11]$ for details. In other words, there always exists a nonsingular matrix $T$ such that

$H_{1, W_{\gamma}}=T^{-1} \operatorname{blockdiag}\left(H_{\gamma, W_{s}}, A_{d},-A_{d}\right) T$,

with $\left(s I-A_{d}\right)^{-1} \in H\left(m_{d}\right)$. This means that the poles of $m_{d}$ are eigenvalues of $H_{1, W_{\gamma}}$, i.e., the assumption of the lemma is also almost always violated. In practice, we can circumvent this problem by slightly altering $V$ and obtain upper and lower bounds for the optimal value [6].

In what follows, we derive a Hamiltonian-based formula for the optimal mixed sensitivity computation, i.e., the problem of finding the maximal $\gamma$ such that 1 is a singular value of $W_{c}$.

\section{Main result}

Consider the singular value equation

$y=W_{c} x, \quad x=W_{c}^{*} y$,

where $W_{c}^{*}$ is the adjoint operator of $W_{c}$. Let $\left(A_{\gamma}, B_{\gamma}, C_{\gamma}\right)$ be a minimal realization of $W_{\gamma}$. Following exactly the same argument in [14, Proposition 2.6], we can show that these equations are characterized by finite dimensional vectors as follows:

$y=W_{\gamma} x-m(s) C_{\gamma}\left(s I-A_{\gamma}\right)^{-1} \xi$,

$x=W_{\gamma} \tilde{y}+B_{\gamma}\left(s I+A_{\gamma}^{\mathrm{T}}\right)^{-1} \zeta$,

where $\xi, \zeta \in \mathbb{R}^{n+p}$ and $n$ and $p$ are the degrees of $W_{s}$ and $m_{d}$, respectively. Combining these equations together, and following the same argument as given in [14], we obtain the following Hamiltonian-based characterization:
Lemma 3. Under the definitions above, 1 is a singular value of $W_{c}$ if and only if there exists a nonzero vector $\left[\xi^{\mathrm{T}} \zeta^{\mathrm{T}}\right]^{\mathrm{T}} \in \mathbb{R}^{2(n+p)}$ such that

$\Phi(s):=\left(s I-H_{1, W_{\gamma}}\right)^{-1}\left[\begin{array}{c}m(s) \xi \\ \zeta\end{array}\right] \in H(m)$.

By invoking the Dunford integral, we can reduce this lemma to a rank condition [14]. Partition $T$ accordingly to (13),

$\left[\begin{array}{ll}T_{11} & T_{12} \\ T_{21} & T_{22} \\ T_{31} & T_{32}\end{array}\right]:=T$,

where $T_{11}, T_{12} \in \mathbb{R}^{2 n \times(n+p)}$ and other four matrices are in $\mathbb{R}^{p \times(n+p)}$. We are now ready to give a formula for the optimal mixed sensitivity for stable plants.

Theorem 4. Define the matrices $H_{1, W_{\gamma}}, H_{\gamma, W_{s}}$ and $T_{i j}(i=1,2,3, j=1,2)$ by (1), (13) and (15). Suppose that $m$ is analytic on the set of the eigenvalues of $H_{\gamma, W_{s}}$. Then the optimal mixed sensitivity $\gamma_{\mathrm{opt}}$ in (6) is the maximal $\gamma$ such that

$\left[\begin{array}{cc}T_{11} & m_{v} \tilde{v}\left(H_{\gamma, W_{s}}\right) T_{12} \\ T_{21} & 0 \\ 0 & T_{32}\end{array}\right]$

is not of full rank.

Proof. From Lemma 3, it suffices to show that there exists a nonzero vector $\left[\begin{array}{ll}\xi^{\mathrm{T}} & \zeta^{\mathrm{T}}\end{array}\right]^{\mathrm{T}} \in \mathbb{R}^{2(n+p)}$ satisfying (14) if and only if the matrix in (16) is not of full rank.

Since $T$ is nonsingular, $\Phi(s)$ belongs to $H(m)$ if and only if so does $T \Phi(s)$, or equivalently,

$$
\begin{aligned}
& \left(s I-H_{\gamma, W_{s}}\right)^{-1}\left[\begin{array}{ll}
T_{11} & T_{12}
\end{array}\right]\left[\begin{array}{c}
m(s) \xi \\
\zeta
\end{array}\right] \in H(m), \\
& \left(s I-A_{d}\right)^{-1}\left[\begin{array}{ll}
T_{21} & T_{22}
\end{array}\right]\left[\begin{array}{c}
m(s) \xi \\
\zeta
\end{array}\right] \in H(m)
\end{aligned}
$$

and,

$$
\left(s I+A_{d}\right)^{-1}\left[\begin{array}{ll}
T_{31} & T_{32}
\end{array}\right]\left[\begin{array}{c}
m(s) \xi \\
\zeta
\end{array}\right] \in H(m) .
$$

First consider (17). Let $\Delta$ be a closed rectifiable contour that encircles all eigenvalues of $H_{\gamma, W_{s}}$, but none of the singularities of $\tilde{m}$. Since $\tilde{m}$ is analytic at 
eigenvalues of $H_{\gamma, W_{s}}$, this is possible. Consider now the integral

$\frac{1}{2 \pi j} \int_{\Delta}\left(s I-H_{\gamma, W_{s}}\right)^{-1}\left[\begin{array}{ll}T_{11} & T_{12}\end{array}\right]\left[\begin{array}{c}\xi \\ \tilde{m}(s) \zeta\end{array}\right] \mathrm{d} s$.

Notice that, by spectral integral theory [1], this integral equals

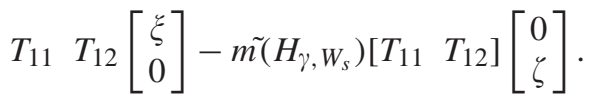

Since (17) holds if and only if this integral is equal to 0, [14], we obtain

$T_{11} \xi=\tilde{m(}\left(H_{\gamma, W_{s}}\right) T_{12} \zeta$.

We now consider condition (18). Recall that we have $\left(s I-A_{d}\right)^{-1} T_{22} \zeta \in H\left(m_{d}\right) \subset H(m)$. Hence in view of (3), (18) is equivalent to $\left(s I-A_{d}\right)^{-1} T_{21} \xi \in H_{-}^{2}$. Since $A_{d}$ has no unstable eigenvalues, this implies

$T_{21} \xi=0$.

For (19), we have $m(s)\left(s I+A_{d}\right)^{-1} \in m_{v} H\left(m_{d}\right) \subset$ $H(m)$ and all eigenvalues of $-A_{d}$ are unstable. Therefore we must have

$T_{32} \zeta=0$.

Combining (20)-(22) together yields

$$
\left[\begin{array}{cc}
T_{11} & m_{v}\left(H_{\gamma, W_{s}}\right) T_{12} \\
T_{21} & 0 \\
0 & T_{32}
\end{array}\right]\left[\begin{array}{l}
\xi \\
\zeta
\end{array}\right]=0 .
$$

There exists a nonzero $\left[\begin{array}{ll}\xi^{\mathrm{T}} & \zeta^{\mathrm{T}}\end{array}\right]^{\mathrm{T}}$ satisfying (23) if and only if the matrix in (16) is not of full rank. This completes the proof.

Remark 5. When $W_{t}=0$, this problem becomes the sensitivity optimization, and $\left[\begin{array}{ll}T_{11} & T_{12}\end{array}\right]=I$ and $p=0$. In this case, we can verify that the rank condition in Theorem 4 is equivalent to $\left.m_{v} \tilde{v}\left(H_{\gamma, W_{s}}\right)\right|_{22}$ is not of full rank, which is the generalized Zhou-Khargonekar formula for the one-block case as expected.

\section{Example}

Suppose that the weighting functions are given by $W_{s}(s)=1 /(s+1)$ and $W_{t}(s)=(s+0.5) /(s+1)$, and a

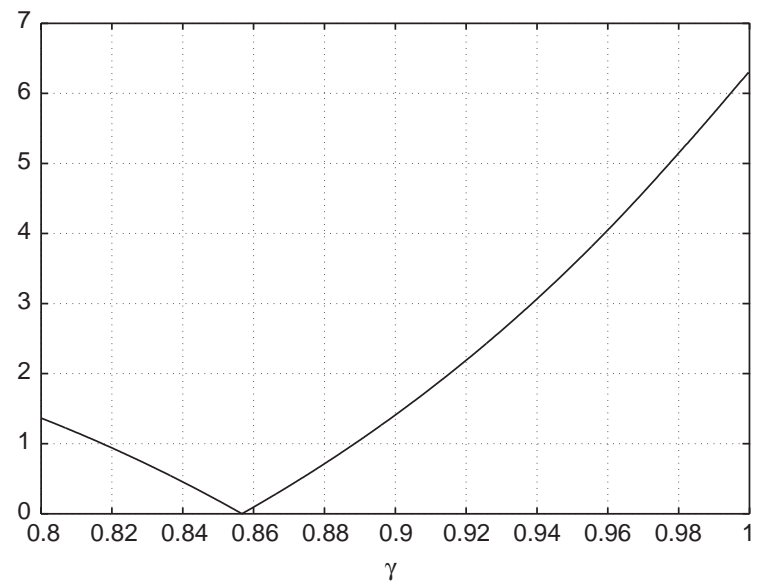

Fig. 1. $\sigma_{\min }$ versus $\gamma$.

stable pseudorational plant $P(s)=\left(\mathrm{e}^{s}-2\right) /\left(2 \mathrm{e}^{2 s}-1\right) \in$ $H^{\infty}$. Then the function $m_{v}$ in (5) is given by

$m_{v}:=\mathrm{e}^{-s} \frac{2 \mathrm{e}^{-s}-1}{2-\mathrm{e}^{-s}}$.

Then, by (7), (8) and (10), $m_{d}, V$ and $W$ are given by

$m_{d}(s)=\frac{s+\lambda}{s-\lambda}, \quad V(s)=\frac{1}{(s+1)(s-\lambda)}$,

$W(s)=\frac{s+0.5}{(s+1)(s-\lambda)}$,

where $\lambda=-\sqrt{5} / 2$. We see that $V$ and $W$ have common poles. Function $W_{\gamma}$ is given by

$W_{\gamma}=\frac{1}{\gamma\left(s^{2}+b s+a\right)}$,

where

$a=\frac{\sqrt{5-\gamma^{-2}}}{2}$ and $b=\sqrt{\frac{9}{4}+2 a-\gamma^{-2}}$.

The eigenvalues of $H_{1, W_{\gamma}}$ are $s= \pm \lambda, \pm \sqrt{1-\gamma^{-2}}$, including the pole of $m_{d}$.

In [6], by changing the weighting function $W_{\gamma}$ slightly, it has been shown that $0.852<\gamma_{\text {opt }}<0.857$. Fig. 1 shows the smallest singular values of the matrix in (16) versus $\gamma$. According to Theorem 4, the optimal mixed sensitivity $\gamma_{\text {opt }}$, the maximal $\gamma$ such that this minimal singular value equals to zero, is approximately 0.8567 and this satisfies the estimate above. 


\section{Conclusions}

We have derived a new closed form Hamiltonianbased formula to the optimal mixed sensitivity optimization problem for stable pseudorational plants with rational weights. This result can be viewed as an extension of the Zhou-Khargonekar formula to a specifically structured one-block problem.

\section{Appendix. Constraint on the derived weighting functions}

Here we see the structure of weighting functions, when we reduce the mixed sensitivity optimization problem to the two-block problem (9) or the problem of finding the singular values of the compression operator $W_{c}$. Consider rational weighting functions $W_{s}=n_{s} / d_{s}, \quad W_{t}=n_{t} / d_{t}$ where pairs of polynomials $\left(d_{s}, n_{s}\right)$ and $\left(d_{t}, n_{t}\right)$ are coprime. For simplicity, we assume that $d_{s}$ and $d_{t}$ have no common zeros. Let us take a stable polynomial $d_{G}$ such that

$$
d_{G} d_{G}=n_{s} n_{s} d_{t} d_{t}+n_{t} n_{t} d_{s} d_{s} \text {. }
$$

Then we have $G=d_{s} d_{t} / d_{G}$ and $m_{d}=d_{G} / d_{G}$. Hence weighting functions in the two-block problem (9) are given by $W=n_{s} n_{s} \tilde{d} \tilde{t} / d_{s} d_{G}$ and $V=n_{s} n_{t} / d_{G}$, and have common poles. Now let us define a stable polynomial $d_{F}$ such that

$d_{F} d_{F}=\gamma^{2} d_{G} d_{G}-n_{s} n_{s} n_{t} n_{t}$.

The spectral factor $F_{\gamma}$ in (12) is given by $F_{\gamma}=d_{G} / d_{F}$, and its zeros are poles of $m_{d}$.

\section{References}

[1] N. Dunford, J.T. Schwartz, Linear Operators, Parts I-III, Interscience, New York, 1963.
[2] C. Foias, H. Özbay, A. Tannenbaum, Robust Control of Infinite Dimensional Systems, Lecture Notes in Control and Information Sciences, Springer, Berlin, vol. 209, 1996.

[3] P.A. Fuhrmann, On the Hamiltonian structure in the computation of singular values for a class of Hankel operators, in: Mosca, Pandol (Eds.), $H_{\infty}$-Control Theory, Lecture Notes in Mathematics, vol. 1496, 1991, pp. 250-276.

[4] K. Hirata, Y. Yamamoto, A. Tannenbaum, A Hamiltonianbased solution to the two block $H^{\infty}$ problem for general plants in $H^{\infty}$ and rational weights, Systems Control Lett. 40 (2000) 83-95.

[5] K. Hoffman, Banach Spaces of Analytic Functions, PrenticeHall, Englewood Cliffs, NJ, 1962.

[6] K. Kashima, H. Özbay, Y. Yamamoto, On the mixed sensitivity optimization problem for stable pseudorational plants, Proceedings of the Fourth IFAC Workshop on Time Delay Systems, INRIA Rocquencourt, France, 2003.

[7] K. Kashima, Y. Yamamoto, A new characterization of invariant subspaces of $H^{2}$ and applications to the optimal sensitivity problem, Systems Control Lett., 54 (2005) 539-545.

[8] T.A. Lypchuk, M.C. Smith, A. Tannenbaum, Weighted sensitivity minimization: General plants in $H^{\infty}$ and rational weights, Linear Algebra Appl. 109 (1988) 71-90.

[9] L. Schwartz, Théorie des Distributions, Herman, Paris, 1966.

[10] M.C. Smith, Singular values and vectors of a class of Hankel operators, Systems Control Lett. 12 (1989) 301-308.

[11] O. Toker, H. Özbay, $H^{\infty}$ optimal and suboptimal controllers for infinite dimensional SISO plants, IEEE Trans. Automat. Control 40 (1995) 751-755.

[12] Y. Yamamoto, Pseudorational input-output maps and their realizations: a fractional representation approach to infinitedimensional systems, SIAM J. Control Optim. 26 (1988) $1415-1430$.

[13] Y. Yamamoto, Equivalence of internal and external stability for a class of distributed systems, Math. Control Signals Systems 4 (1991) 391-409.

[14] Y. Yamamoto, K. Hirata, A. Tannenbaum, Some remarks on Hamiltonians and the infinite-dimensional one block $H^{\infty}$ problem, Systems Control Lett. 29 (1996) 111-117.

[15] K. Zhou, P.P. Khargonekar, On the weighted sensitivity minimization problem for delay systems, Systems Control Lett. 8 (1987) 307-320. 